\title{
The LimSee3 Multimedia Authoring Model
}

\author{
Romain Deltour \\ INRIA Rhône-Alpes \\ 655 avenue de l'Europe \\ 38334 Saint Ismier, France \\ Romain.Deltour@inria.fr
}

\author{
Cécile Roisin \\ UPMF, INRIA Rhône-Alpes \\ 655 avenue de l'Europe \\ 38334 Saint Ismier, France \\ Cecile.Roisin@inria.fr
}

\begin{abstract}
For most users, authoring multimedia documents remains a complex task. One solution to deal with this problem is to provide template-based authoring tools but with the drawback of limited functionality. In this paper we propose a document model dedicated to the creation of authoring tools using templates while keeping rich composition capabilities. It is based on a component oriented approach integrating homogeneously logical, time and spatial structures. Templates are defined as constraints on these structures.
\end{abstract}

\section{Categories and Subject Descriptors}

I.7 [Document and Text Processing]: Document Preparation-Hypertext/hypermedia, Multi/mixed media, Standards

\section{General Terms}

Design, Languages

\section{Keywords}

multimedia documents, document models, document authoring, template-based editing

\section{INTRODUCTION}

The LimSee3 project aims at defining a document model dedicated to adaptive and evolutive multimedia authoring tools, for different categories of authors and applications, in order to easily generate documents in standard formats. It is a follow-up to LimSee2 (a SMIL authoring tool) and is related to our involvement in the Urakawa project [5].

Our experience with LimSee2 has convinced us that direct manipulation of well-established languages such as SMIL (or $\mathrm{XMT}$ ) is too complex for most users because it requires a deep understanding of the semantics of the language (e.g. the SMIL timing model). That is why we are looking in LimSee3 for an independent authoring model. From the user perspective a document is logically structured in several high

Permission to make digital or hard copies of all or part of this work for personal or classroom use is granted without fee provided that copies are not made or distributed for profit or commercial advantage and that copies bear this notice and the full citation on the first page. To copy otherwise, to republish, to post on servers or to redistribute to lists, requires prior specific permission and/or a fee.

DocEng'06, October 10-13, 2006, Amsterdam, The Netherlands.

Copyright 2006 ACM 1-59593-515-0/06/0010 ...\$5.00. level objects, but in SMIL these are distributed in several parts of the document (spatial information in the header, time information in the body). Several software tools [1], [2], [6] hide the intrinsic complexity of SMIL behind advanced GUI or by adding dedicated information via namespaced attributes. However, SMIL is too low level for this kind of tools to be completely satisfactory. Hence we are also looking at template-based solutions such as [3] or [7].

In this paper we propose to define a model for these templates. Our approach is to focus on the logical structure of the document while keeping some semantics of proven technologies such as SMIL. This provides better modularity, facilitates the definition of document templates, and improves manipulation and reusability of content.

\section{APPROACH}

Existing structured multimedia models generally put the time structure at the heart of the multimedia document. It was for instance the case of CMIF [10] and Madeus [8], as well as SMIL. However, the time dimension does not always reflect exactly the logical structure in the way it is considered by the author. This latter logical structure is made of at least both time and spatial dimensions, which are specified in SMIL in distinct sections of the document. Our approach defines the logical dimension as the master structure of the document, which is a tree of modular components that can be constrained by a dedicated template mechanism.

A template document is a kind of reusable document skeleton that provides a starting point to create document instances. Domain specific template systems are a user-friendly authoring solution but require hardly extensible dedicated transformation process to output the rendering format. We chose on the contrary to tightly integrate the template syntax in the document: the template is itself a document constrained by schema-like syntax. The continuum between both template and document permits to edit templates generically as any other document and within the same environment. It enables an evolutive authoring of document instances from templates. There is no need to define a dedicated language to adapt to each different use case.

With these objectives, we define a structured authoring language independently of any publication language. Elements of the master structure are components that represent semantically significant objects. Both time and spatial dimensions are integrated inside each component. This permits components to be authored independently, integrated in the document structure, extracted for reusability, constrained by templates or referenced by other components. 
While truly modular, this component approach raises the issues of inter-object relations and extraction of components from their context. The different components of a multimedia document are indeed often tightly related one with another: when they are synchronized, when they are aligned in space, when one contains an interactive link to the other, and so on. Our approach, which is close to the one proposed in [11] is for each component to abstract its dependencies to external components by giving them symbolic names that are used in the timing and layout sections. This abstraction layer facilitates the extraction of a component from its context, thus enhancing modularity.

One might think that this component-based approach provides less reusability of the layout definition as in SMIL, but the layout is still reusable in the scope of the component. Actually, the major drawback is that global views of the document (global timing scenario, global spatial layout) are not directly accessible but need to be computed, which might be costly. This is more an implementation issue though.

Finally, the goal was to rely on proven existing technologies, in both contexts of authoring environments and multimedia representation. The timing and positioning models are wholly taken from SMIL. Using XML provides excellent structuring properties and enables the use of many related technologies. Among them are XPath, used to provide finegrained access to components, and XSLT, used in templates for structural transformation and content generation.

\section{THE AUTHORING LANGUAGE}

The authoring language is twofold: it consists in a generic document model for the representation of multimedia documents, and it defines a dedicated syntax to represent templates for these documents.

\subsection{Document Model}

A document is no more than a document element wrapping the root of the object hierarchy and a head element containing metadata. This greatly facilitates the insertion of the content of a document in a tree of objects, or the extraction of a document from a sub-tree of objects.

A compound object is a tree structure composed of nested objects. Each compound object is defined by the object element with the type attribute set to compound. It contains a children element that lists children objects, a timing element that describes its timing scenario and a layout element that describes its spatial layout.

The value of the required localId attribute uniquely identifies the component in the scope of its parent object, thereby also implicitly defining a global identifier id when associated with the localId of the ancestors. In Example 1, the first child of object parent has the local id child1 and hence is globally identified as parent.child1.

The timing model, and similarly the positioning model, are taken from SMIL 2.1. The timing element defines a SMIL time container as specified in the timing and synchronization module of SMIL 2.1. The timing scenario of a component is obtained by composition of the timed inclusions defined by the timeRef elements, whose refId attributes are set to local ids of children.

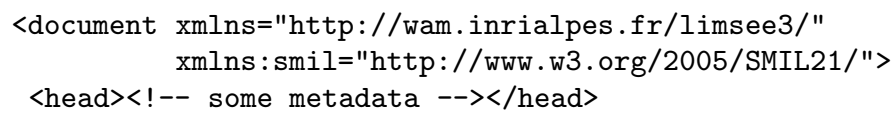

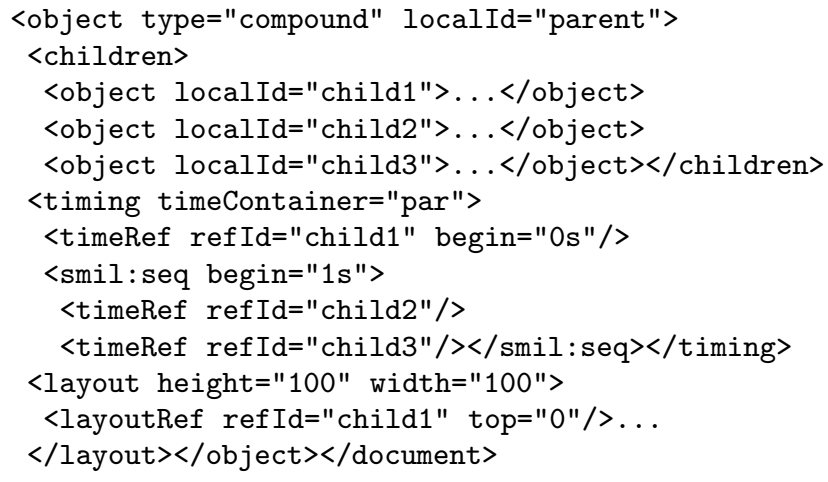

\section{Example 1: A Partial LimSee3 Document}

A media object is actually a simple object that wraps a media asset, i.e. an external resource (such as an image, a video, an audio track, a text...) referenced by its URI. It is defined by the object element with the type attribute set to either text, image, audio, video or animation (this list can be extended in the future). The URI of the wrapped media asset is the value of the src attribute. Example 2 shows an image media object with local id linkImage which wraps the media asset identified by the URI ./medias/image.jpg.

Area objects inspired from the SMIL area element can be associated with media objects. They are used for instance to structure the content of a media object or to add a timed link to a media object. An area is defined as an object element with the type attribute set to area. For instance, in example 2 the image media object linkImage has a child area which defines a hyperlink.

Relations of dependency between objects are described independently of their semantics in the document. External dependencies are declared with ref elements grouped inside the related child element of objects. The value of refId of a ref element is the id of the related element and the value of localld is a symbolic name that is used within the object to refer to the related object. For instance, in Example 2, object linkImage describes an image that links to the object extObj1, by first declaring the relation in a ref element and then using this external object named target to set the value of the href attribute of the link, using attribute and value-of elements taken from XSLT.

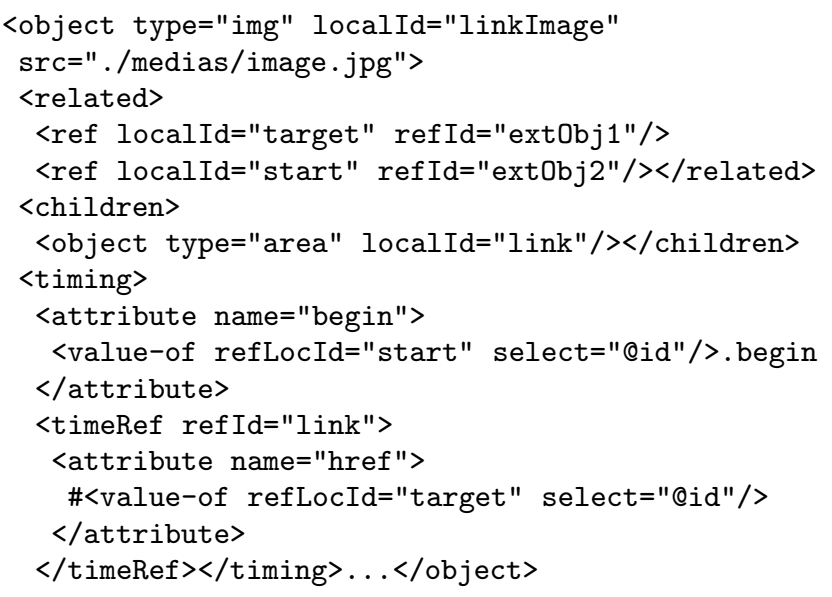

Example 2: A LimSee3 Object with External Dependency Relations 


\subsection{Templates}

Template nodes aim at guiding and constraining the edition of the document. In order to have better control and easy GUI set up, the language defines two template nodes: media zone and repeatable structure.

A media zone is a template node that defines a reserved place for a typed media object. It is represented by the zone element with a type attribute (text, img, audio, video, animation, any, or a list of these types). The author of the template can also specify content that will be displayed to invite the user to edit the media zone with the invite element (of any media type). For instance Example 3 shows a media zone for an image, with textual invitation. During the authoring process zone elements aim at being filled by media objects inserted by the user.

A repeatable structure, represented by the objList element, is a template node that defines a homogeneous list of object. Each item of the list matches a model object declared in the model child of the objList. The cardinality of the list can be specified with the min0ccurs and max0ccurs attributes. Example 3 shows a simple slideshow as an objList named list and containing image media objects as specified by the model slide.

It is possible to lock parts of a document with the locked attribute, to prevent the author from editing anything. This permits for instance to guide more strongly inexperienced users by restricting their access to the only parts of the document that make sense to them.

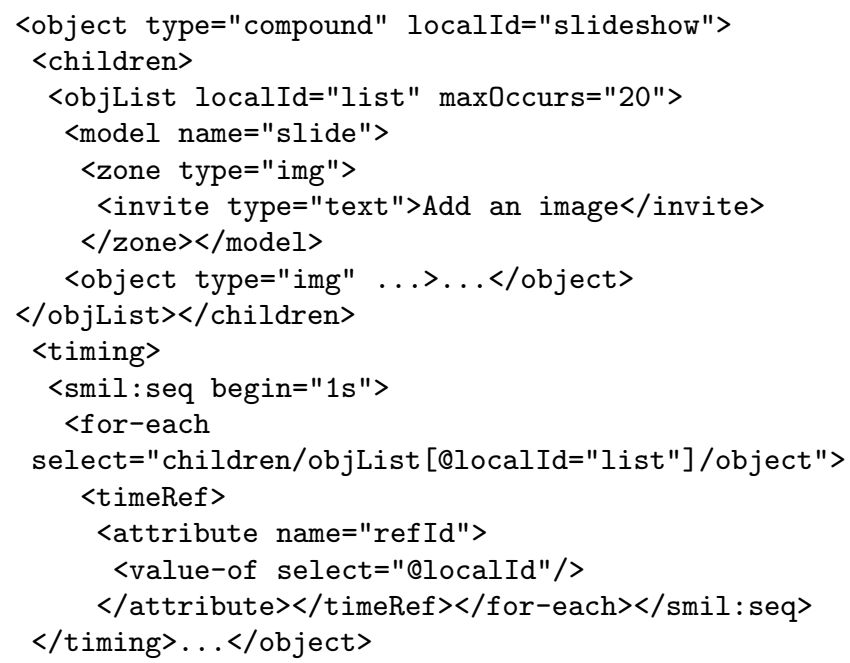

Example 3 : A Simple Slideshow Template

\section{THE AUTHORING PROCESS}

The tight integration of template nodes in the document model ensures a continuous authoring workflow. As shown in Figure 1, a document is progressively instantiated from a template by providing content to template nodes (for instance, in Example 3 the object list list is partially instantiated) ; a template can conversely be authored starting from a document instance. Once fully authored, a document can be exported to any target format, provided the semantics of this latter is included in our document model. These basic authoring services (instance edition, template intantiation, publication) directly rely on the LimSee3 document model. They are being implemented as a cross-platform java soft- ware on which dedicated authoring tools with appropriate user-friendly GUI can be built.

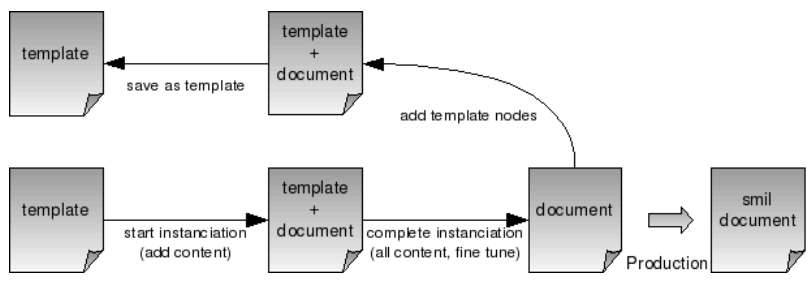

Figure 1: The Authoring Process

\section{CONCLUSION}

The model presented in this paper improves reusability not only with template definitions but also by the homogeneous structuring of documents. The homogeneous use of components (for instance in XPath expressions) facilitates the extensibility of the language and the evolution of existing documents. The generic authoring platform is on the way to be completed and new authoring tools will be developed mainly in the context of the European project PALETTE [4] for pedagogical CoPs. The declarative and modular approach of LimSee3 model paves the way for providing extensions such as authoring adapted documents or defining behavioral reactivity in documents as proposed by $[9]$.

\section{REFERENCES}

[1] IBM Resarch: Authoring in XMT. http://www.research.ibm.com/ mpeg4/Projects/AuthoringXMT/.

[2] LimSee2. http://wam.inrialpes.fr/software/limsee2/.

[3] Microsoft Producer for PowerPoint. http://www.microsoft.com/ office/powerpoint/producer/prodinfo/.

[4] Palette. http://palette.ercim.org/.

[5] Urakawa Project. http://www.daisy.org/projects/urakawa/.

[6] D. Bulterman and L. Hardman. Structured multimedia authoring. ACM TOMCCAP, 1(1), 2005.

[7] X. Hua, Z. Wang, and S. Li. Lazycut: Content-aware template based video authoring. In $A C M$ Multimedia'05. ACM Press, 2005.

[8] M. Jourdan, N. Layaïda, C. Roisin, L. Sabry-Ismaïl, and L. Tardif. Madeus, an authoring environment for interactive multimedia documents. In $A C M$ Multimedia'98. ACM Press, 1998.

[9] P. King, P. Schmitz, and S. Thompson. Behavioral reactivity and real time programming in $\mathrm{xml}$ : functional programming meets smil animation. In ACM DocEng'04. ACM Press, 2004.

[10] L.Hardman, G. van Rossum, and D. Bulterman. Structured multimedia authoring. In $A C M$ Multimedia'93. ACM Press, 1993.

[11] H. Silva, R. Rodrigues, L. Soares, and D. M. Saade. $\mathrm{Ncl}$ 2.0: integrating new concepts to xml modular languages. In ACM DocEng'04. ACM Press, 2004. 